\title{
Technology-Delivered Intervention Strategies to Bolster HIV Testing
}

\author{
Romina A. Romero ${ }^{1}$ • Jeffrey D. Klausner ${ }^{2}$ - Lisa A. Marsch ${ }^{3}$ - Sean D. Young ${ }^{1,4}$ (D) \\ Accepted: 18 May 2021 / Published online: 9 June 2021 \\ (C) The Author(s), under exclusive licence to Springer Science+Business Media, LLC, part of Springer Nature 2021
}

\begin{abstract}
Since the beginning of the HIV epidemic, there have been more than 75 million cases. Currently, there about 1.2 million living with HIV in the USA. Despite current testing recommendations, test rates continue to be suboptimal. Investigators have studied the use of digital technology to promote HIV testing, especially among high-risk populations.
\end{abstract}

\section{Purpose of Review}

This non-systematic review provides an overview of the scientific research between 2015 and 2020 focused on the use of digital technology to bolster HIV testing and suggests novel technologies for exploration.

\section{Recent Findings}

A total of 40 studies were included in the review that span a wide range of available technology. Studies effectively increased HIV testing among study participants. Generally, participants in the intervention/exposure groups had significantly higher rates of HIV test uptake compared to participants in the comparison groups at study follow-up.

\section{Summary}

For a variety of reasons (e.g., differences in ways the technologies were used and study design), no digital tool clearly performed better than others, but each have the capacity to increase outreach and self-testing. An exploration of the potential use of nascent technologies is also discussed, as well as the authors' experiences using a number of these technologies in our research.

Keywords HIV $\cdot$ Self-testing $\cdot$ Digital tools $\cdot$ Technology

This article is part of the Topical Collection on Behavioral-Bio-Medical Interface

Sean D. Young

syoung5@hs.uci.edu

1 Department of Emergency Medicine, University of California, Irvine, Irvine, CA, USA

2 Division of Disease Prevention, Policy and Global Health, Department of Preventive Medicine, University of Southern California Keck School of Medicine, Los Angeles, CA, USA

3 Geisel School of Medicine, Dartmouth College, Hanover, NH, USA

4 Department of Informatics, University of California, Irvine, 6091 Bren Hall, Irvine, CA 92617, USA

\section{Introduction}

Increasing rates of HIV testing is one of the most important strategies in reducing the burden of HIV worldwide. Current estimates of prevalence report 38 million adults and children worldwide are diagnosed with HIV [1]. In the USA, 1.2 million Americans are currently living with HIV, and despite testing availability and accessibility, an estimated $14 \%$ are unaware of their infection [2]. Current US Centers for Disease Control and Prevention recommendations suggest testing all patients in healthcare settings ages 13 to 64 years at least once, with those at higher risk testing annually [3]. However, two-thirds of those at high risk for infection who visited a healthcare provider in the past year were not offered an HIV test [4]. Using electronic health records, investigators found that only $6.4 \%$ of Americans over 18 years had any laboratory evidence of a prior HIV test [5]. These findings fall 
far short of the United Nations' 90-90-90 treatment goal that by $2020,90 \%$ of all people living with HIV will know their status, $90 \%$ of all people with diagnosed infection will receive sustained antiretroviral treatment (ART), and $90 \%$ of all people receiving ART will achieve viral suppression [6]. Investigators estimate that $40 \%$ of transmission during the non-acute phase is due to those unaware of their status [7]. This highlights the importance of testing, as individuals who become aware of their infection are likely to modify their risk behaviors $[8,9]$.

Digital technologies, such as social media, mobile and virtual reality applications (apps), chatbots and conversational agents, and blockchain technology, have the potential to increase HIV testing. Novel approaches that leverage digital technologies may be particularly helpful in efforts to reach high-risk populations [10]. Trend data indicate an upward pattern in social media use [11] and smartphone ownership [12] in most demographics, including African Americans and Hispanics. In addition, historically, gay, lesbian, and bisexual adults were earlier adopters of technology compared to their heterosexual counterparts $[13,14]$. A similar increasing trajectory is seen in mobile app use [15], virtual reality market size [16], and smart speaker ownership [17]. Researchers have capitalized on the ubiquity and variety of technology to increase HIV testing. For instance, investigators found that social media interventions aimed at promoting HIV testing among men who have sex with men (MSM) were effective, and that test uptake was higher for intervention groups vs. controls [18, 19]. Similarly, investigators examining the effects of mobile health (online and smartphone apps) found increased HIV testing $[19,20]$ and decreased condomless anal intercourse [19, 20].

This manuscript seeks to provide an overview of the science behind technologies that can be incorporated to increase HIV testing. We focus specifically on publications published in the last 5 years, for this special issue. We have organized results of these publications in categories based on the specific type of technology they use. Technologies included are as follows: social media, study website, live chat, text messages, mobile applications, online videos, electronic health records, vending machines, conversational agents, virtual reality, and the potential use of blockchain technology and mobile payment services to increase HIV testing.

\section{Methods}

We conducted a non-systematic review of various technologies used in HIV testing research, designed to inform the reader of example technologies that have been leveraged to promote HIV testing, along with study results (when studies had been conducted with this type of technology). Many of these types of technologies were used in combination with one another in HIV testing research (e.g., social media and data websites/apps were used to recruit participants to visit a website created specifically for the study). Therefore, the categories were not designed to be mutually exclusive but rather to provide the reader with examples of various types of technological approaches that might be incorporated into future research and clinical applications.

Three electronic reference databases (PubMed, Google Scholar, Web of Science) were searched for this nonsystematic review using keywords and Medical Subject Headings for studies published between 2015 and 2020 . Search terms included the following: (1) HIV testing, (2) digital tools, (3) social media, (4) chatbot, (5) conversational agent, (6) electronic health records, (7) vending machines, (8) blockchain, (9) virtual reality, (10) self-testing, (11) men who have sex with men, and (12) MSM. We included men who have sex with men and MSM in our key terms due to their high risk of HIV infection, though this review is not limited to this population but focuses on publications about technology used in HIV prevention research. We included completed studies as well as manuscripts that describe protocols of ongoing studies. A total of 40 studies were selected from the search that were representative of a variety of categories included in this non-systematic review. In addition, recent research conducted by study authors of relevance to the review was included.

\section{Results}

\section{Social Media}

Social media includes any digital tool used to create content and share ideas, opinions, and information with a virtual network of people and communities [21]. Research in the past 5 years suggests social media can be an effective tool for increasing HIV testing among high-risk populations, including MSM [18, 22, 23], as well as using "social big data" to monitor HIV-related outcomes [24-30].

Posts and advertisements placed on social media have been shown to be effective in encouraging social media users to request HIV test kits. For example, in a randomized control trial (RCT), Rhodes et al. engaged with MSM on social media sites (Adam4Adam, BlackGayChat, Craigslist, and Gay. com.) via a public profile created specifically for the study. Posts included information about the importance of HIV testing, testing locations, and the process to access tests. After the 12-month intervention, participants in the intervention communities had higher rates of testing for HIV compared to the comparison communities who did not receive any information (AOR 2.9, 95\% CI 1.8-4.7) [31]. Lemley et al. utilized paid advertisements on three different types of social media platforms (social media sites, dating sites/apps, 
informational sites) to examine the effectiveness of the platforms to promote HIV testing among racial/ethnic minority MSM. Study recruitment ended in July 2020 and investigators will follow up with participants at two time points to compare the performance of each platform in promoting and encouraging HIV testing among users [32]. Washington and colleagues posted short videos on the intervention group social media page weekly and encouraged study participants (young, black MSM) to engage with the page. Compared to control participants who received weekly standard informative text posts (no videos), intervention participants were more likely to get tested (OR 7.0, 95\% CI, 1.72, 28.33, p=0.006) [33].

Social media intervention studies have also included engaging study participants using peer leaders (i.e., MSM trained in how to reach out to other MSM peers on social media to increase HIV testing). For example, a study was efficacious in increasing uptake of HIV testing among MSM living in Peru. Participants were recruited using social mediatargeted banner advertisements. Trained peer leaders engaged with intervention participants in private Facebook groups. Compared to the control group (no peer models in the Facebook groups), more participants in the intervention group engaged with the group and received an HIV test at a local community clinic (AOR 2.61, 95\% CI 1.55-4.38) [34]. Young and colleagues recently completed a similar 5-year among minority MSM in Greater Los Angeles designed to increase HIV self-test requests. They have begun analyzing primary outcome with promising results. Similarly, in a randomized comparative trial, Patel and colleagues used peer models to engage participants on social media, a mobile app, or email based on participant preference and to deliver twomessage framing strategies to promote HIV testing: (1) desirable outcome to be achieved and (2) undesirable outcome to be avoided. In addition, participants from both groups had access to a study website that offered free HIV test kits, condoms, and a list of services for sexually transmitted infections. Results showed that regardless of which message framing the participants were exposed to, testing increased between baseline and follow-up among participants (31.5 to 43.8\%, $\mathrm{p}=0.04$ ) [35]. Table 1 shows a brief summary of each study.

\section{Study Website}

Another effective communication tool to provide information about HIV and the importance of testing is a study-specific website (i.e., a website created specifically for a research study and designed to direct participants directly to it). Several investigators launched study websites with general information about HIV. Boni et al. offered free HIV self-test kits using a study website. The website was promoted via flyers at outreach events and gay- and MSM-friendly venues, as well as virtual messages on social media and posts on dating apps. The study website had 67,225 page views with 17,786 unique visitors over the study period. A total of 3885 requests for HIV self-test kits were made and 2526 were delivered. Of those delivered, $21 \%$ of participants reported results, suggesting that an internet-based HIV self-test kit approach was feasible in the study population [36]. MacGowan and colleagues found similar results to Boni et al. in that offering free tests through study websites increased HIV testing. Intervention and control participants were given a link to the study website which provided HIV information and prevention, but intervention participants received free self-test kits that the control group did not. Intervention participants tested more frequently than control participants, and more had tested at least three times by the 12-month follow-up compared to controls $(76.6 \%$ vs. $22.0 \%$, $\mathrm{p}<0.01)$ [41]. Bauermeister and colleagues developed and provided a link to a personalized website for intervention participants that utilized theory-based sessions to promote positive sexual health and HIV testing compared to the attentiononly controls who received information only. The website contained six sessions which included a core message, indepth discussion of topics related to the core message, and interactive activities. Activities and videos presented were designed to build participant HIV risk reduction skills. Although the website was highly accepted among the participants, it did not increase testing at follow-up compared to controls who were provided a website with general HIV prevention information $\left(46.7 \%\right.$ vs. $\left.40.0 \%, X^{2}(1)=0.49, p=0.49\right)$ [43]. Increasingly, instead of using study-specific websites that require a new type of website to be built for each study, researchers are beginning to use "research recruitment" websites that allow researchers to post their studies online and assist in recruiting participants to those studies using their outreach and participant databases. For example, ElevateU's website, Moshemu (https://moshemu.com/) is a National Institute on Drug Abuse-funded website designed to help researchers identify participants for substance use and HIV-related studies (and to help potential participants find studies).

\section{Live Chat with Human Staff}

Live chat with humans during at home testing may facilitate future testing. Two studies examined the efficacy of chatting with study staff while taking a self-test at home in promoting future testing. Wang et al. found that intervention participants who watched a 7-min video and received a live video chat with study staff while taking a home self-test reported higher rates of testing at a 6-month follow-up compared to control participants who watched a 3-min online video only $(89.8 \%$ vs. $50.7 \%$, RR $1.77, \mathrm{p}<0.001$ ) [38]. Similarly, in a study looking at the efficacy of a home-based test paired with a video-chat counseling session during test administration, Stephenson and colleagues noted that all control participants (home-based self-test kit only) ordered self-test kits and reported their results to the study website. Of the 126 
Table 1 Study characteristics by technology used

\begin{tabular}{|c|c|c|c|}
\hline Author & Study type & Participants & Study results \\
\hline
\end{tabular}

Social media

Patel et al., $2020[26]$

Randomized comparative trial to study feasibility, acceptability, and impact of peer-delivered messaging for HIV testing and consistent condom use. Two groups: (1) approach (desirable outcome to be achieved) group and (2) avoidance (undesirable outcome to be avoided) group.

Rhodes et al., Randomized community trial to increase HIV 2016 [22] testing through posts on social media. Two groups: (1) two intervention communities received information about HIV testing information and (2) two control communities did not receive any information.

Washington RCT to examine feasibility of video intervention on et al., 2017 Facebook to increase HIV testing. Two groups: [24] (1) intervention group viewed five 1-minute videos weekly and (2) control group viewed standard HIV-related text information only.

Young et al., RCT to examine the efficacy of peer models who 2015 [25] engaged participants on Facebook to increase request for HIV test. Two groups: (1) intervention groups with peer models and (2) control groups without peer models.

Study websites

Bauermeister et al., 2019

[29]

RCT to determine the acceptability and efficacy of an online intervention to change sexual risk behaviors and HIV testing. Two groups: (1) intervention group received distinct and affective content designed to build HIV risk reduction skills and positive sexual health behaviors and (2) control group provided with general HIV prevention content.

Boni et al., Intervention study to examine the feasibility of an 2019 [27] internet-based HIV self-test kit approach to increase HIV testing.

MacGowan

et al., 2020

[28]

Live chat

Stephenson et al., 2020

[31]

RCT to evaluate the effect of providing HIV self-test kits on testing frequency, diagnoses of infection, and sexual risk behaviors. All participants received information through study website. Two groups: (1) intervention group which received a self-test kit in the mail and (2) control group which did not receive a kit.

RCT to test an online HIV prevention intervention using telehealth and home-based HIV self-test kit. Two groups: (1) intervention group received an HIV self-test kit and an appointment with a remote counselor who supervised administration of the test and (2) control group that was mailed one HIV self-testing kit.

Wang et al., 2018 [30]

RCT to examine the efficacy of an online counseling session during a home-based HIV 18+-year-old MSM

18-30-year-old Black MSM

18+-year-old MSM

18-24-year-old gay, bisexual, and MSM

18+-year-old MSM

18+-year-old MSM

15-24-year-old transgender youth

18+-year-old MSM test. Two groups: (1) intervention group received the same video as control plus an additional
OR for 6-week post intervention HIV testing 7.0 (95\% CI, 1.72, 28.33, $p=0.006$ ).

Increased self-reported HIV testing from baseline to 6-months follow-up (31.5 to $43.8 \%$, $\mathrm{P}=0.04)$. No significant difference in condom use between baseline and follow up. HIV testing and condom use did not differ between two different intervention groups.

Post-test HIV testing for intervention group was $63.7 \%$ compared to $42.0 \%$ for comparison group (AOR 2.9, 95\% CI 1.8-4.7).

HIV testing for intervention group was $17.0 \%$ compared to $7.0 \%$ for control group (AOR $2.61,95 \%$ CI $1.55-4.38$ ).

$45 \%$ of participants reported testing for HIV at 3-month follow-up, with no differences observed between the two groups ( $46.7 \%$ vs. $40.0 \% ; X[2](1)=0.49, \mathrm{p}=0.49)$.

The study website garnered 67,225 page views with 17,786 unique visitors during the study period. A total of $2526 \mathrm{HIV}$ self-test kits were delivered and $21 \%$ of participants reported results online or by mail.

HIV testing for intervention group at least 3 or more times during the trial was $76.6 \%$ compared to $22.0 \%$ for control group $(\mathrm{P}<0.01)$.

65 participants randomized into the intervention group did not participate in the intervention. Of the remaining intervention participants, HIV testing during the trial was $96 \%$ compared to 91\% for control group. At 3-months follow-up, STI testing for intervention was $72.5 \%$ compared to $42.9 \%$ for the control group ( $\mathrm{p}=$ 0.001 ), but declined by 6-months follow-up for both groups (intervention arm 19.2\%, control arm $15.3 \%)$.

HIV testing of any test type for intervention group was $89.8 \%$ compared to $50.7 \%$ for control group at 6-month follow-up (RR 1.77, $\mathrm{p}<0.001$ ). 
Table 1 (continued)

\begin{tabular}{|c|c|c|}
\hline Author & Study type & Participants \\
\hline
\end{tabular}

Short message service/text message

video, an HIV self-test kit, a video chat

counseling session and (2) control group

received a 3-minute online HIV prevention video

and encouragement to tested.

Kelvin,

RCT to test the efficacy of a text message reminder for a self-test HIV kit. Three groups: (1)

George,

Kinyanjui,

et al., 2019

[35] intervention group received three text messages about the new self-test HIV kit, (2) enhanced standard of care group received the standard text message reminder for a finger-prick test administered at the clinic three times, and (3) standard of care group received the standard text message once.

Kelvin, $\quad$ RCT to test the efficacy of a text message reminder George, for a self-test HIV kit. Three groups: (1)

Mwai, et al., intervention group received three text messages 2019 [36] about the new self-test HIV kit, (2) enhanced standard of care group received the standard text message reminder for a finger-prick test administered at the clinic three times, and (3) standard of care group received the standard text message once.

Njuguna et al., Quasi-experimental study to examine the effect of 2016 [33] text messages in increasing HIV testing. Two groups: (1) intervention group received weekly HIV-related text messages and (2) control group did not receive text messages.

Wettermann Proof-of-concept study to examine the feasibility of et al., 2019 a text message campaign to encourage patients to [32] facilitate HIV testing discussion with their physician and increase HIV testing. Two groups: (1) group received HIV testing text message and (2) group received vaccine or calcium intake text message (control message).

Ybarra et al., RCT to test the effect of a text messaging-based

2017 [34] HIV prevention program. Two groups: (1) intervention group that received HIV informational text messages and (2) control group that received healthy lifestyle text messages.

Mobile/dating application

Balán et al., Multi-phase study to develop and test a 2020 [37] smartphone-based app to increase HIV testing among users and their partners.

Horvath et al., RCT to test the feasibility, acceptability, and impact 18+-year-old MSM $2020[38]$ of a mobile app to increase HIV testing. Two groups: (1) intervention group had access to a mobile app with HIV-related information and (2) no treatment control group.

Pai et al., 2018 Cohort to investigate if mobile app increased [39] 18+-year-old male truckers (driver or assistant)

18+-year-old female sex workers

18-24-year-old college women

14-18-year-old cisgender male who identified as gay, bisexual, and/or queer

18+-year-old MSM or transgender women who have sex with men
Patients from primary care clinics
HIV testing during the study period for the intervention group was $3.5 \%$ compared to $1.3 \%$ for the enhanced standard of care group (OR 2.7, 95\% CI 1.3-5.5). No difference was found in testing rates for enhanced standard of care group and standard of care group groups.

HIV testing during the study period for the intervention group was $10.8 \%$ compared to $6.1 \%$ for the enhanced standard of care group (OR 1.9, 95\% CI 1.3-2.7). No difference was found in testing rates for enhanced standard of care and standard of care groups.

HIV testing at the 6-month follow-up for the intervention group was $67 \%$ compared to $51 \%$ for the control group (hazard ratio $1.57,95 \% \mathrm{CI}$ $1.28-1.92, \mathrm{p}<0.001)$.

$35 \%$ of the group who received an HIV text message requested a test and none of the patients who received the control message requested an HIV test.

HIV testing post intervention for the intervention group that was sexually active at baseline was $38.9 \%$ compared to $18.1 \%$ for the control group (AOR 3.39, p<0.001). At 90-day follow-up, testing for the intervention group was $55.1 \%$ compared to $28.2 \%$ for the control group (AOR 3.42, p<0.001).

Participants found the app useful and engaging. Most watched the video tutorial before self-testing and scanned their test results with the app.

Overall, the app was feasible and well accepted by participants. At 4-months, repeat HIV testing for the intervention group was $17 \%$ compared to $4 \%$ of the control group. However, at 8 months, repeat HIV testing for the intervention group was $50 \%$ compared with $40 \%$ of the control group.

Referrals for self-testing was $17.4 \%$ compared to control group $2.6 \%$ (RR 6.69, 95\% CI 4.47-10.01) and many new infections 86 vs 57 (OR 1.55, 95\% CI 1.1-2.2). linkages to care. Three groups: (1) supervised self-testing, (2) unsupervised self-testing, (3) control with conventional testing. 
Table 1 (continued)

\begin{tabular}{lll}
\hline Author & Study type & Participants \\
\hline $\begin{array}{l}\text { Rosengren } \\
\text { et al., 2016 }\end{array}$ & $\begin{array}{c}\text { Intervention study to examine the feasibility of } \\
\text { banner ads placed in a dating app (Grindr) to } \\
\text { [40] }\end{array}$ & $\begin{array}{c}\text { 18+-year-old Black and } \\
\text { distribute HIV self-test kits. Ads linked to a study } \\
\text { website where participants were able to order a }\end{array}$ \\
& $\begin{array}{l}\text { test kit. } \\
\end{array}$
\end{tabular}

Sullivan et al., Intervention study to examine the acceptability and 18+-year-old MSM 2017 [41] usability of an Android-based HIV prevention app.

\author{
Study results
}

Banner ads placed on Grindr were effective in reaching high-risk populations. The study website received 4389 unique visitors and 333 test requests. Participants were open to providing personal information to obtain a free test kit, which participants found acceptable and easy to use.

Participants used the app for an average of 17.7 min over the study period. App users ordered condoms $(63.3 \%)$ and HIV self-test kits $(52.8 \%)$ through the app. During the study period, $77 \%$ of participants tested for HIV at least once and 56\% tested multiple times. Participant thought the app was easy to use and $69 \%$ stated they would download the app again.

Wray et al., 2018 [42]

RCT to increase HIV testing, post-test follow-up care, and referral. Three groups: (1) eTEST group that was mailed a self-test kit in packaging attached to a device that alerted study investigators when the package was opened to initiate a follow-up call from a counselor, (2) standard group which received a self-test kit with no follow-up call, and (3) control group that was mailed informational letters about HIV testing lefts.

Zhu et al., 2019 [43]
RCT to test a mobile-based app designed to increase 18+-year-old MSM HIV self-testing. Two groups: (1) intervention group received two HIV self-test kits and access to study app that provided messages, videos, and referrals to health services related to HIV and (2) control group received two HIV self-test kits only. 18+-year-old MSM

HIV testing for eTEST and standard groups during the study period was $100 \%$ compared to $72 \%$ for the control group $(\mathrm{p}=0.001)$. There were no significant differences in testing behavior between the two groups that received self-test kits. Repeat testing was also higher for participant in these two groups compared to control participants (81\% eTEST, $77.2 \%$ standard vs. $40.9 \%, \mathrm{p}<0.001)$. However, the eTEST group was significantly more likely to receive risk reduction counseling, prevention supplies, and PrEP referrals compared with the other two groups.

HIV testing in the past 6 months at follow-up for intervention group was $92 \%$ compared to $68 \%$ for the control group $(\mathrm{p}<0.001)$. Intervention group significantly increased

HIV testing behaviors in the past 6 months compared to controls (ARR $=1.99,95 \% \mathrm{CI}$ $1.03-3.84, \mathrm{p}<0.05)$ and use of oral HIV self-test $(\mathrm{ARR}=2.17,95 \%$ CI $1.08-4.37, p<$ 0.05 )

Informational videos

Aronson et al., Intervention study to examine the feasibility and 2016 [44] acceptability of a tablet-based video designed to address barriers to HIV testing in the Emergency Department.

Aronson et al., Intervention study to examine the feasibility and 2017 [45] acceptability of a tablet-based video designed to provide opioid overdose prevention and response and facilitate HIV and HCV testing in community settings.

Tang et al.,

Non-inferiority RCT to compare first time HIV self-test rates among participants who viewed HIV test promotion videos. Two groups: (1) group that received crowdsourced HIV test video 18-24-year old Emergency Department patients

18+-year-old clients of a community-based syringe exchange program

16+-year-old MSM and transgender individuals
Of the 100 participants enrolled in the study, $98 \%$ completed the intervention (mean time $<9$ mins) and 30\% accepted HIV tests offered by the tablets. Post-test showed significantly higher knowledge after than before the intervention $(\mathrm{t}=-6.67, \mathrm{p}<.001)$. Overall, patients found the intervention highly acceptable, easy to use, and understandable.

Of the 11 participants enrolled in the HIV module, 91\% accepted HIV tests offered by the tablet. All participants in the HCV module accepted the HCV test, but only $60 \%$ of the overdose prevention module agreed to receive additional naloxone training. Overall, participants in the three different modules accepted the intervention well with acceptability scores no less than 6.8 out of 10 .

HIV testing for the crowdsourced group was $37 \%$ compared to $35 \%$ for the health marketing group. The estimated difference between the groups was $2.1 \%$ (95\% CI -5.4 to $9.7 \%$ ). 
Table 1 (continued)

\begin{tabular}{ll}
\hline Author & Study type \\
\hline & $\begin{array}{l}\text { and (2) group that received health marketing HIV } \\
\text { test promotion video. }\end{array}$ \\
& \\
Electronic health record \\
Ahlström & Investigators examined the feasibility of using \\
et al., 2019 & electronic registry data to predict HIV status \\
[47] & using machine learning algorithms.
\end{tabular}

\section{Kershaw Retrospective cohort analysis of electronic medical et al., 2018 records before and after the implementation of a [48] reminder in the electronic medical record for HIV screening.}

Marcus et al., Investigators examined the feasibility of using 2019 [49] electronic registry data to predict incident HIV diagnosis using absolute shrinkage and selection operator logistic regression to identify potential candidates for PrEP.

Sha et al., Investigators examined the feasibility of a screening 2019 [50] program of electronic health records to increase HIV testing, diagnosis, and linkage to care in the emergency department.

Vending machine

He et al., 2019 Intervention to examine the acceptability,

[51] feasibility, and effectiveness of a vending machine that dispensed HIV urine specimen collection kit for HIV testing.

Lee et al., 2020 [52]

Cross-sectional structured survey to identify acceptability, preferences, and concerns about HIV test kits dispensed from vending machines.

Stafylis et al., Intervention study to increase HIV testing through 2018 [53] delivery of free HIV self-test kits dispensed in vending machines.

Vera et al., 2019 [54]

Participants

Study results

Members of a national hospital registry who met study criteria and at least 16 years old

18-65-year-old patients of a medical left

18+-year-old patients of a medical left who met

13-64-year-old patients of a medical left who met study criteria

University students

6+-year-old Black African, Black Caribbean, and Latin American study criteria

Investigators conclude that crowdsourcing may be a feasible tool for effective HIV test promotion messaging.

Machine learning algorithms can learn from electronic health records to predict HIV cases and distinguish them from non-HIV cases with high accuracy. Further integration of prediction models may be complementary to condition-guided HIV testing and identification of patients suitable for PrEP.

Overall, HIV testing increased to $30.7 \%$ after the reminder, up from $15.3 \%$ (RR 2.02, CI 1.95-2.09, $\mathrm{p}<0.0001)$. The significant increase $(\mathrm{p}<0.0001)$ was seen in all age groups.

Prediction models using electronic health record data were able to identify incident HIV cases. Simpler models identified fewer patients and had lower sensitivity for incident HIV cases compared to the full model (6.0-28.9\% vs. $38.6 \%$ of incident HIV cases).

Overall, HIV testing increased to $17.1 \%$ after electronic health record screening was implemented and $21.1 \%$ when HIV testing was linked to blood test orders, up from $2.5 \%$ at baseline.

11 vending machines were placed in seven universities during an AIDS/STD educational campaign. Of 957 urine collection kits distributed by the campaign during the study period, 197 were sold through the vending machines and 760 were freely distributed. $64.5 \%$ of kits purchased from the vending machines were returned for analysis. $72.2 \%$ of kits purchased were from vending machines in dormitories and $27.8 \%$ from instructional buildings.

Rates of willingness to use HIV self-test kits from vending machines were high in all groups except Black Caribbeans (Black Africans 77.9\%, Black Caribbeans 53.6\%, Latin Americans 81.8\%, other non-white ethnicities $80.0 \%$ ). Participants preferred vending machines located in healthcare settings over community settings.

Vending machines were placed in two commercial sex venues in the on-site STI testing room and social lounge. A total of 1398 kits were dispensed through vending machines, and 110 surveys were completed by patrons who obtained a free test kit. Of those surveyed and reported that the kit was used, 95\% reported using the test kit themselves, and $17.7 \%$ of these participants reported a reactive result.

18+-year-old clients of a sauna frequented by MSM
A total of 265 test kits were dispensed through the vending machine. HIV testing was 34 tests per month through the vending machine compared acceptability of vending machines to dispense HIV self-test kits in community settings. Two 
Table 1 (continued)

\begin{tabular}{|c|c|c|c|}
\hline Author & Study type & Participants & Study results \\
\hline & $\begin{array}{l}\text { comparative methods: }(1) \text { test kits dispensed } \\
\text { through vending machines and (2) test kits } \\
\text { distributed through community workers. }\end{array}$ & & $\begin{array}{l}\text { with } 6 \text { tests a month through the outreach } \\
\text { testing by community workers in the same } \\
\text { venue. The vending machines were accepted } \\
\text { by the participants with } 94 \% \text { of survey } \\
\text { respondents stating they would use the vending } \\
\text { machine or recommend it to others. }\end{array}$ \\
\hline $\begin{array}{c}\text { Young et al., } \\
2014 \text { [55] }\end{array}$ & $\begin{array}{l}\text { Qualitative study to examine the usability and } \\
\text { acceptability of using vending machines to } \\
\text { dispense HIV self-test kit. }\end{array}$ & $\begin{array}{l}\text { 18+-year-old African } \\
\text { American and Latino } \\
\text { MSM }\end{array}$ & $\begin{array}{l}\text { Overall, participants interviewed stated that } \\
\text { obtaining an HIV self-test kit from a vending } \\
\text { machine was acceptable. Participants differed } \\
\text { in opinion regarding aesthetics of the vending } \\
\text { machine but agreed that confidentiality and } \\
\text { customization made using a vending machine } \\
\text { acceptable. Participants felt confident that the } \\
\text { test kit they obtained from the vending machine } \\
\text { was an accurate medical device and sanitary. } \\
\text { Other factors for acceptability of using a test kit } \\
\text { from a vending machine included: ease of use, } \\
\text { time efficiency, and confidentiality. }\end{array}$ \\
\hline \multicolumn{4}{|c|}{ Chatbots/conversational agents } \\
\hline $\begin{array}{c}\text { Brixey et al., } \\
2017 \text { [56] }\end{array}$ & $\begin{array}{l}\text { Implementation paper on designing a chatbot using } \\
\text { Facebook application programming interface } \\
\text { within its Messenger platform. }\end{array}$ & & $\begin{array}{l}\text { Developers used a variety of credible sources to } \\
\text { create a body of questions and answers about } \\
\text { HIV/AIDS which served as the foundation of } \\
\text { knowledge for the chatbot. Developers used a } \\
\text { response classifier and dialogue management } \\
\text { system to drive responses to questions. }\end{array}$ \\
\hline $\begin{array}{l}\text { van Heerden } \\
\text { et al., } 2017 \\
\text { [57] }\end{array}$ & $\begin{array}{l}\text { Experimental study to explore the feasibility of } \\
\text { using a conversational agent embedded in a } \\
\text { messaging mobile application to provide pre-test } \\
\text { counseling prior to an HIV test. }\end{array}$ & & $\begin{array}{l}\text { Six of the } 10 \text { testers preferred a quick reply button } \\
\text { instead of typing out responses to the chatbot } \\
\text { and thought that both the aesthetics and } \\
\text { appropriate responses made the interaction feel } \\
\text { like a conversation with a human. Other testers } \\
\text { thought the interaction was not realistic, and } \\
\text { that the language was too formal. Seven testers } \\
\text { felt that the interaction was private and } \\
\text { anonymous and therefore, they would be } \\
\text { willing to take a test with the chatbot. }\end{array}$ \\
\hline $\begin{array}{l}\text { Vermey et al., } \\
2019 \text { [58] }\end{array}$ & $\begin{array}{l}\text { Assessment of an online advice application with a } \\
\text { chatbot feature that provides tailored advice } \\
\text { based on user-provided information regarding } \\
\text { personal characteristics, sexual behavior, sexual } \\
\text { risk, and symptoms. }\end{array}$ & & $\begin{array}{l}\text { The online application was visited } 337,736 \text { times } \\
\text { in } 2018 \text { with } 113,257 \text { visitors started the } \\
\text { questionnaire, and } 17,449 \text { utilizing the chatbot. } \\
\text { Around } 60 \% \text { of visitors finished the } \\
\text { questionnaire, which led to } 65,736 \text { advice } \\
\text { given, and } 8700 \text { clicks to online self-test } \\
\text { providers. }\end{array}$ \\
\hline
\end{tabular}

$R C T$ randomized control trial, $M S M$ men who have sex with men, $P r E P$ pre-exposure prophylaxis, (A)OR (adjusted)odds ratio, $C I$ confidence interval, (A)RR (adjusted)relative risk, $H C V$ hepatitis $\mathrm{C}$ virus

participants randomized to the intervention group (homebased self-test kit plus one-time video-chat counseling), only 61 ordered test kits and participated in the video-chat counseling session; two took the self-test prior to the scheduled counseling appointment. At 3-months follow-up, sexually transmitted infections (STI) testing was significantly higher in the intervention group than control $(72.5 \%$ vs. $42.9 \%, \mathrm{p}=$ $0.001)$, but was not significant at the 6-month follow-up $(19.2 \%$ vs. $15.3 \%)$. The investigators concluded that programs should recognize the diverse needs of participants since not all may be willing to take an HIV self-test during a live chat [37].

\section{Short Message Service/Text Messages}

Text messages are a quick and convenient method to send HIV-related information to study participants. Several investigators used various messages to promote uptake of HIV testing. Wettermann et al. compared the number of HIV test orders by physicians between intervention and control groups. Thirty minutes prior to the participant's scheduled medical appointment, those in the intervention group received a message about HIV prompting them to discuss HIV with their physician while the control group received a message about a general health topic. Approximately 35\% of intervention 
participants requested a test, while none of the control group requested one [39]. Other types of messages were successful in increasing uptake of HIV testing among respective target populations. Investigators texted messages about HIV prevention and reproductive health [42], HIV information and testing, behavioral skills, relationship and coming out [59], and availability of self-test kits available at health clinics [40, 60]. Regardless of message type, text messages resulted in increases in testing among study participants.

\section{Mobile Apps and Dating Apps}

Investigators have also used apps and programs on mobile devices such as smartphones to educate about HIV and promote HIV self-testing in high-risk groups. Sullivan and colleagues examined the acceptability of a theory-based mobile phone app among MSM. Study results showed that over half of participants used the app to order condoms (63\%) and HIV self-test kits (52.8\%) [44]. Similarly, a study of an intervention to increase HIV testing delivered via a popular mobile app (WeChat) was conducted. Intervention group participants received two oral HIV self-test kits and access to the app which provided HIV information and referrals to health services, while the control group received two test kits only. Investigators found increased testing rates among those who received the intervention compared to the control group (92\% vs. $68 \%, p<0.001$ ) [45]. Horvath et al. examined the feasibility and acceptability of an HIV testing reminder app (intervention group) among MSM to increase repeat testing. Findings showed that although more participants in the intervention group (17\%) repeatedly tested at the four month mark compared to the control group (4\%; no treatment), the difference between the two arms dissipated by the eighth month (50\% vs. $40 \%$ ) [46]. Investigators examined the feasibility and acceptability of a smartphone app that provided HIV information, test video instructions, and information on how to interpret test results. Self-test was conducted using a custom-built smartphone accessory or a rapid test [48], with or without supervision at various locations [50]. Participant feedback was positive for the feasibility and usability of the app. Wray et al. conducted a pilot three-arm randomized control trial to increase testing in a high-risk population. Two groups received test kits, with one group receiving kits equipped with devices and a follow-up call once the test kit is opened (eTEST) and the other group a standard kit with no followup call (standard). The third group received informative letters about testing locations and a reminder to get tested every 3 months (control). More participants who received a free home test kit tested at least once during the study period (eTEST and standard) compared to controls $(100 \%$ vs. $72 \%, \mathrm{p}=0.001)$. Repeat testing was also higher for participants in these two groups compared to control participants (81\% eTEST, $77.2 \%$ standard vs. $40.9 \%, \mathrm{p}<0.001)$. Participants who also received a follow-up call after study staff were notified that the test kit was opened received more information about risk reduction counseling, prevention supplies, and PrEP referrals than controls and standard of care participants [49]. Based on the pilot study, Wray and colleagues have begun a longitudinal randomized control trial with an oversampling of African American and Latino MSM. The same three study arms will be used for the trial with a few differences: the control group will receive text messages instead of a letter every 3 months to remind them to get tested at a local clinic [47]. Another use of mobile health is an app that facilitates HIV testing such as Safely. Users can store their most recent test results as well as connect with a testing facility using the app [61]. Paid advertisements on a dating app site specifically for MSM were effective in attracting users to a study website where they can order an HIV test kit [52].

\section{Informational Online Videos}

Three studies utilized short informative videos to promote HIV testing. Aronson and colleagues conducted a pilot program targeting young adult patients in the emergency department (ED) who declined an HIV test at triage. Upon completing the intervention, watching the short video on a tablet, participants increased their knowledge about HIV and 30\% accepted the HIV test offered by the tablet [53]. A similar intervention was tested in a community setting for people who inject drugs. Participants watched a short video on a tablet about HIV, HCV, or overdose prevention while visiting a syringe exchange program facility. Findings showed that participants found the intervention acceptable, increased knowledge after watching the short video, and accepted the HIV test $(91 \%)$, HCV test $(100 \%)$, or naloxone $(60 \%)$ offered by the tablet [51]. Tang and colleagues compared the effect of two videos in increasing uptake of HIV testing in a noninferiority randomized control trial. One video was created using a small marketing company while the other video utilized crowdsourcing for development. Investigators found no significant difference in first time HIV testing between the two groups (37\% vs 35\%), suggesting that crowdsourcing may be a novel approach to creating health messages and engaging the community [54, 62].

\section{Electronic Health Records}

The use of electronic health records (EHR) is another technology that may promote HIV testing. Investigators employed simple techniques to use artificial intelligence to increase HIV testing in the healthcare setting. Kershaw et al. examined the effect of incorporating a reminder in the EHR for HIV antibody to prompt physicians to screen patients. Findings showed a significant increase in screening after a reminder was incorporated for all patients age 18-65 (15.3\% baseline 
vs $30.7 \%$ post reminder, (RR 2.02, CI 1.95-2.09, p < 0.0001) [55]. To enhance HIV testing in the ED, Sha and colleagues screened ED EHRs using specified criteria and "Best Practice Advisory" and then auto ordered screening for complete blood count. Through this process, investigators were able to identify acute seroconverters, new chronically ill patients, and known infected patients (many who did not disclose their status) [56]. Investigators utilized machine learning algorithms that would identify patients at risk for HIV acquisition among adult members of a large healthcare organization [57] and national hospital registry [58]. In both studies, various models were tested to discriminate and correctly predict between HIV cases and non-cases.

\section{Vending Machines}

The use of vending machines to dispense HIV self-test kits has been shown to be acceptable and feasible in increasing testing. Investigators have found that dispensing HIV self-test kits in vending machines was acceptable among MSM [63] and blacks/African American, Hispanic/Latino, and other minorities [64]. Placement of vending machines may be particularly key in reaching populations at most risk. Stafylis and colleagues placed vending machines in commercial sex venues which dispensed 1398 test kits. Of kits that were used, 17.7\% reported a reactive result [65]. He et al. placed vending machines with urine-based HIV self-test kits at universities. Forty percent of dispensed kits were submitted for testing, of which less than $2 \%$ were positive [66]. Vera and colleagues compared the amount of HIV test kits dispensed by a vending machine to that delivered by community workers. Vending machines placed in a sauna (British gay bathhouse) dispensed more HIV self-test kits (34 kits per month) than were delivered by community workers (6 test kits per month) [67]. Preferences for vending machine location and test kit placement varied among adult MSM; some preferred the vending machine to be customized to reflect that it was dispensing HIV self-test kits, while others preferred the test kits to be integrated with other items (i.e., snacks) to increase confidentiality [68].

\section{Chatbot/Conversational Agents}

Chatbot and conversational agents to promote HIV testing use natural learning processing to allow for real-time dialogue with artificial intelligence designed to answer questions about HIV and testing. Chatbot interfaces use text and audio to interact with users. For instance, Brixey and colleagues developed a chatbot using a response classifier and dialogue management system on a Facebook application programming interface that was programmed with information from state and federal public health agencies to populate responses to user questions. Once live, users will be able to engage with the text chatbot via Facebook Messenger [69]. A conversational agent designed to provide HIV pre-test counseling was tested for technical feasibility and acceptance among users. Participants were provided a mobile phone with the app to facilitate discussion. Study findings indicated that users had a positive experience and that the interaction with the agent felt natural. Privacy, anonymity, and convenience of the chatbot compared to in-person methods were cited as benefits [70]. Vermey and colleagues developed a chatbot within an online advice website that provided personalized advice. Some of the site visitors used the chatbot features $(17,449$ visitors) and clicked on online self-test providers ( 8700 clicks) [71]. Aside from research purposes, chatbots are used by an international HIV/AIDS organization to provide information about HIV to youth. Users have responded positively to the chatbot stating that the chatbot was "cool" and praised the developers for the project [72]. A potential use of virtual assistant applications (i.e., Alexa, Siri) in promoting HIV testing is to provide pre-test counseling, a directory of online vendors of home test kits, or nearby locations where users can get tested. As information seeking tops the list of requests for virtual assistants [73], they can be programmed with information aimed to encourage testing. The ability of a user to have a dialogue with the virtual assistants may minimize some of the perceived barriers such as stigma to get tested. Another use of the virtual assistants is placement of HIV test kits in a shopping cart or subscription service. Similar to a chatbot for opioid use disorder (OUD) currently in development by one of the co-authors of this paper (Young), a conversational agent that would facilitate locating HIV testing sites could be beneficial for communities at high risk for infection. The OUD chatbot aims to identify an appropriate treatment facility for those misusing opioids. Targeted for patients and family, the chatbot would engage users in dialogue until a facility that suits the needs of the patient can be identified.

\section{Virtual Reality}

Several investigators have used virtual reality (VR) in HIV research, which may have applications in promoting HIV testing. In one study, participants who used a VR scenario to practice HIV status disclosure to a partner said the simulations were easy to use, they would use it frequently, and most people would learn to use the system easily [74]. In another study, investigators examined the effect of VR on increasing antiretroviral therapy adherence. Study findings showed participant experience resulted in decreased viral load and increased CD4(+). Participants stated the experience was comfortable, educational, and that it would help increase adherence [75]. VR scenarios can be developed to help increase self-efficacy in discussing STI testing with sexual partners or with a healthcare provider. VR games can provide various situations and environments during which relevant conversations will 
most likely occur to afford users with ample opportunities to respond, learn, and gain confidence.

\section{Blockchain Technology}

Nascent technology such as blockchain may facilitate promotion of HIV/STI testing. Blockchain is an immutable public ledger [76]; a publicly accessible electronic data storage that cannot be altered even by its owner. It is the underlying technology that runs cryptocurrency and has since been utilized in industries such as healthcare, ecommerce, and finance [77]. To date and to our knowledge, no study exists that examines the use of blockchain technology with respect to HIV research; however, some of its features may be applicable to increasing uptake of HIV testing. A smart contract, which is a contract between two parties without the need of a third party (i.e., public notary) to be executed [78], may be beneficial to users of social networking apps. For instance, after initial engagement through a dating app, both users can upload their current test results to blockchain as proof of testing. Users would meet only after blockchain has verified that both users have uploaded their test results. Another feature of the blockchain technology that may be useful in increasing HIV testing is the hybrid blockchain. A hybrid blockchain is managed by a network of stakeholders (i.e., healthcare provider, testing facility, insurance company), instead of the public or one central organization, that affords the owner of the blockchain a high degree of control of who can access the data [79]. This would allow patients to have access to their test results at their convenience and can facilitate testing conversation with potential sex partner before intercourse. In terms of cryptocurrency, payment using cryptocurrency may appeal to potential test takers. Researcher may also use cryptocurrency, or combine with gamification as reward, to pay for HIV self-test kits.

\section{Mobile Payment Services}

Alternative payment, such as mobile payment services, may incentivize HIV testing. Though no study to our knowledge has examined the effect of type of payment in obtaining HIV tests, the convenience of paying vendors through services such as Venmo, Apple Pay, or Zelle may attract those who prefer contactless payment. Trend data show increasing use of digital payment, and in the past year, even surpassed that of credit card and debit card use [80]. Merchants from all industries are reimagining their operating and payment models to accommodate the dramatic changes in on-demand movement [81]. The ease of using mobile payment services may appeal to those purchasing home self-test kits via online vendors, vending machines, or mobile apps.

\section{Ethical and Implementation Considerations}

Ethical concerns may affect the impetus to obtain an HIV test promoted through digital technology [82]. Despite some potential ethical concerns, participants in a social media study to increase HIV testing expressed that they felt comfortable participating in an online social media study and that they benefitted from participation [82]. Another consideration when dealing with participants with potential medical diagnosis is the willingness of participants to share digital data with researchers. Romero and Young conducted a study that examined the willingness of patients with diagnosed medical conditions to share private data with researchers. Participants voiced concerns over privacy and confidentiality when determining how much data to provide scientific researchers. Though some patients expressed concerns about data storage, who will access the data, and if data will be used against them, most reported being comfortable or very comfortable sharing social media, GPS location, and electronic medical records with researchers who would be attempting to use the data for public health purposes [83]. As the field of applying digital technology to promote HIV testing continues to evolve, it is key that a parallel, priority focus embraces important scientific questions related to ethics and privacy to understand the array of related factors that may impact real-world adoption. To support this goal, ethics committees should be composed of members who are experts or knowledgeable of digital tools and can provide guidance in the appropriate use of technology for research and specific ethical considerations that warrant examination.

Researchers using technology to increase HIV testing may have additional implementation considerations to safeguard participant safety and engagement. Investigator and study staff should clearly indicate in the consent form what types of data (i.e., location, images, text messages with study staff) are being collected by the technology (i.e., app, smartwatch, wearable device) and what data are not being collected (i.e., contacts, phone calls, web browsing). An additional layer of transparency may obviate hesitation from potential participants and assuage any concerns about privacy and confidentiality risks. In recruiting participants for research using technology, one barrier to participation may be lack of access to technology necessary to participate in the study. Some devices and applications may only work with certain operating systems that may alienate potential participants who own devices that cannot support those systems. It would behoove investigators to supply the device to participants who may not be eligible otherwise. Lastly, as self-test kits come in different forms, a focus group with the target population might inform investigators of the type of home-based self-test kit that they prefer to use. Though participants will have their own preferences, some test kits are simpler to administer and less invasive than others. 
Worldwide, the COVID-19 pandemic has resulted in significant decreases in HIV testing [1] and impacted the capacity of service providers to meet the needs of the community. This has underscored the importance of and ability to leverage technology in promoting testing and other HIV research and clinical services [84]. The flexibility and convenience of obtaining home test kits via vending machines or through trusted websites may offer alternatives to in-person testing when appointments are limited due to facility closures or limited office hours. The option of chatting with a provider on a smartphone for pre-test counseling or to assist in test administration may alleviate anxiety in taking a home test when inperson consultation is not available. Finally, when implementing technologies into HIV research, it is important to not rely solely on the technology but to ensure the use of technology is grounded in proper design and an evidencedbased model to improve effectiveness $[85,86]$.

\section{Conclusion}

Technologies continue to play an increasing and exciting role in HIV testing. Every new technology presents HIV researchers with an opportunity to explore whether this new tool might be used to increase HIV testing. It is important to note from the categories of studies in this manuscript that no particular type of technology appears to be consistently better or worse than other technologies. Rather, technologies are simply tools that can be used to increase reach, scalability, and efficiency. To properly use technologies to increase HIV testing, researchers need to choose the appropriate technology for the context and population, integrate that technology with an evidenced-based intervention, and have a plan for how to adapt to the changing trends and contexts in use of technologies [85, 87]. In addition, they should also consider the equity and digital divide, and how these interventions might facilitate increases in testing among the digital literati but also might miss some key populations such that more traditional and/or other innovative programs are still needed.

As technology continues to evolve, HIV researchers have incorporated digital tools for outreach, education, and promotion of HIV testing. The success of tools such social media, mobile apps, vending machines, and virtual reality have paved the way for nascent technology such as blockchain and virtual assistants to be adopted in the field. Although decades of efforts to mitigate the HIV epidemic have improved outcomes worldwide, much work continues to be needed to increase HIV testing. In a world that is markedly changing because of advances in digital technology, we have the opportunity to creatively embrace these diverse and ever-growing array of tools to identify new ways to increase access to HIV testing across diverse populations at a global level. Such technology affords wide scale use that may address barriers and challenges faced by those who are at most risk for infection while providing a potentially low-cost, scalable tool for preventing the global spread of HIV. Ultimately, it is about enabling increased choice and options such that access to testing becomes normative and ubiquitous and interventions can reach those most disenfranchised.

Author Contribution Jeffrey D. Klausner, Lisa A. Marsch, and Sean D. Young conceptualized the idea for the paper. Romina A. Romero performed the literature search and drafted the initial review. All authors critically revised the work.

Funding Preparation of this manuscript was partially supported by Grant P30DA029926 from the National Institute on Drug Abuse (NIDA), as well as grants from the National Institute of Allergy and Infectious Diseases, and National Institute of Mental Health.

Availability of Data and Material Not applicable

Code Availability Not applicable

\section{Declarations}

Competing Interests The authors declare no competing interests. SY has received gift funding from Facebook, Intel, and consulting funding from ElevateU.

Human and Animal Rights This article does not contain any studies with human or animal subjects performed by any of the authors.

\section{References}

1. UNAIDS. Global HIV \& AIDS statistics - 2020 fact sheet. UNAIDS.org. 2020.https://www.unaids.org/en/resources/factsheet (accessed 13 Oct 2020).

2. HIV.gov. U.S. Statistics. HIV.gov. 2020.https://www.hiv.gov/hivbasics/overview/data-and-trends/statistics (accessed 13 Oct 2020).

3. Branson BM, Handsfield HH, Lampe MA, et al. Revised recommendations for HIV testing of adults, adolescents, and pregnant women in health-care settings. MMWR Morb Mortal Wkly Rep. 2006;55:1-17.

4. Dailey AF, Hoots BE, Hall HI, et al. Vital signs: human immunodeficiency virus testing and diagnosis delays - United States. MMWR Morb Mortal Wkly Rep. 2017;66:1300-6. https://doi.org/ 10.15585/mmwr.mm6647e1.

5. Niforatos JD, Wanta JW, Durbak E, Cavendish J, Yax JA. Assessing the national prevalence of HIV screening in the United States using electronic health record data. Cureus. 2019;11:e5043. https://doi.org/10.7759/cureus.5043.

6. UNAIDS. 90-90-90: An ambitious treatment target to help end the AIDS epidemic. UNAIDS Information Production Unit 2014.

7. Gopalappa C, Farnham PG, Chen Y-H, Sansom SL. Progression and transmission of HIV/AIDS (PATH 2.0). Med Decis Mak. 2017;37:224-33. https://doi.org/10.1177/0272989X16668509.

8. Eaton LA, Kalichman SC. Changes in transmission risk behaviors across stages of HIV disease among people living with HIV/AIDS. $J$ Assoc Nurses AIDS Care. 2009;20:39-49. https://doi.org/10. 1016/j.jana.2008.10.005. 
9. Marks G, Crepaz N, Senterfitt JW, Janssen RS. Meta-analysis of high-risk sexual behavior in persons aware and unaware they are infected with HIV in the United States: implications for HIV prevention programs. J Acquir Immune Defic Syndr. 2005;39:446-53. https://doi.org/10.1097/01.qai.0000151079.33935.79.

10. Estem KS, Catania J, Klausner JD. HIV Self-Testing: a review of current implementation and fidelity. Curr HIV/AIDS Rep. 2016;13: 107-15. https://doi.org/10.1007/s11904-016-0307-y.

11. Pew Research Center. Social Media Fact Sheet. Pew Research Center. 2019.https://www.pewresearch.org/internet/fact-sheet/ social-media/ (accessed 28 Oct 2020).

12. Pew Research Center, Inquiries. Demographics of mobile device ownership and adoption in the United States. Pew Research Center. 2019.https://www.pewresearch.org/internet/fact-sheet/mobile/ (accessed 4 Nov 2020).

13. Wong N, Gullo K, Stafford J. Gays lead non-gays in cell phone use, cable TV \& HDTV viewership. www.harrisinteractive.com (accessed 6 Feb 2021).

14. Brown A Lesbian, gay and bisexual online daters report positive experiences - but also harassment. Pew Research Center. https:// www.pewresearch.org/fact-tank/2020/04/09/lesbian-gay-andbisexual-online-daters-report-positive-experiences-but-alsoharassment/ (accessed 10 Feb 2021).

15. Clement J Mobile app usage - Statistics \& Facts. Statista. 2019.https:/www.statista.com/topics/1002/mobile-app-usage/ (accessed 4 Nov 2020).

16. Tankovska H. Number of voice assistants in use worldwide 20192024. Statista. 2020.https://www.statista.com/statistics/973815/ worldwide-digital-voice-assistant-in-use/ (accessed 29 Oct 2020).

17. Tankovska H. Smart speaker installed base U.S. 2017-2019. Statista. 2020.https://www.statista.com/statistics/794480/usamazon-echo-google-home-installed-base/ (accessed 4 Nov 2020).

18. Cao B, Gupta S, Wang J, Hightow-Weidman LB, Muessig KE, Tang W, et al. Social media interventions to promote HIV testing, linkage, adherence, and retention: systematic review and meta-analysis. J Med Internet Res. 2017;19:e394. https://doi.org/10.2196/ jmir.7997.

19. Xin M, Viswanath K, Li AY-C, CAO W, HU Y, Lau JTF, et al. The effectiveness of electronic health interventions for promoting HIVpreventive behaviors among men who have sex with men: metaanalysis based on an integrative framework of design and implementation features. J Med Internet Res. 2020;22:e15977. https:// doi.org/10.2196/15977.

20. Ko JS, Stafylis C, Klausner JD. Mobile health promotion of human immunodeficiency virus self-testing in the United States. Mhealth. 2020;6:10. https://doi.org/10.21037/mhealth.2019.10.05.

21. Obar JA, Wildman SS. Social media definition and the governance challenge - an introduction to the special issue. Rochester: Social Science Research Network; 2015. https://doi.org/10.2139/ssrn. 2663153.

22. Krueger EA, Chiu CJ, Menacho LA, et al. HIV testing among social media-using Peruvian men who have sex with men: correlates and social context. AIDS Care. 2016;28:1301-5. https://doi. org/10.1080/09540121.2016.1178699.

23. Garett R, Smith J, Young SD. A review of social media technologies across the Global HIV Care Continuum. Curr Opin Psychol. 2016;9:56-66. https://doi.org/10.1016/j.copsyc.2015.10.024.

24. van Heerden A, Young S. Use of social media big data as a novel HIV surveillance tool in South Africa. PLoS One. 2020;15: e0239304. https://doi.org/10.1371/journal.pone.0239304.

25. Young SD, Yu W, Wang W. Toward Automating HIV identification: machine learning for rapid identification of HIV-related social media data. J Acquir Immune Defic Syndr. 2017;74(Suppl 2):S12831. https://doi.org/10.1097/QAI.0000000000001240.

26. Zhang Q, Chai Y, Li X, Young SD, Zhou J. Using internet search data to predict new HIV diagnoses in China: a modelling study.
BMJ Open. 2018;8:e018335. https://doi.org/10.1136/bmjopen2017-018335.

27. Young SD, Zhang Q. Using search engine big data for predicting new HIV diagnoses. PLoS One. 2018;13:e0199527. https://doi.org/ 10.1371/journal.pone.0199527.

28. Young LE, Soliz S, Xu JJ, Young SD. A review of social media analytic tools and their applications to evaluate activity and engagement in online sexual health interventions. Prev Med Rep. 2020;19: 101158. https://doi.org/10.1016/j.pmedr.2020.101158.

29. Zheng C, Zhang Q, Long G, Zhang C, Young SD, Wang W. Measuring time-sensitive and topic-specific influence in social networks with LSTM and self-attention. IEEE Access. 2020;8:8248192. https://doi.org/10.1109/ACCESS.2020.2991683.

30. Young SD. A "big data" approach to HIV epidemiology and prevention. Prev Med. 2015;70:17-8. https://doi.org/10.1016/j.ypmed. 2014.11.002.

31. Rhodes SD, McCoy TP, Tanner AE, et al. Using social media to increase HIV testing among gay and bisexual men, other men who have sex with men, and transgender persons: outcomes from a randomized community trial. Clin Infect Dis. 2016;62:1450-3. https://doi.org/10.1093/cid/ciw127.

32. Lemley SM, Klausner JD, Young SD, Stafylis C, Mulatya C, Oden $\mathrm{N}$, et al. Comparing web-based platforms for promoting HIV selftesting and pre-exposure prophylaxis uptake in high-risk men who have sex with men: protocol for a longitudinal cohort study. JMIR Res Protoc. 2020;9:e20417. https://doi.org/10.2196/20417.

33. Washington TA, Applewhite S, Glenn W. Using Facebook as a platform to direct young black men who have sex with men to a video-based HIV testing intervention: a feasibility study. Urban Social Work. 2017;1:36-52. https://doi.org/10.1891/2474-8684.1. 1.36 .

34. Young SD, Cumberland WG, Nianogo R, Menacho LA, Galea JT, Coates T. The HOPE social media intervention for global HIV prevention in Peru: a cluster randomised controlled trial. Lancet HIV. 2015;2:e27-32. https://doi.org/10.1016/S2352-3018(14) 00006-X.

35. Patel VV, Rawat S, Dange A, Lelutiu-Weinberger C, Golub SA. An internet-based, peer-delivered messaging intervention for HIV testing and condom use among men who have sex with men in India (CHALO!): pilot randomized comparative trial. JMIR Public Health Surveill. 2020;6:e16494. https://doi.org/10.2196/16494.

36. Boni RBD, Veloso VG, Fernandes NM, et al. An internet-based HIV self-testing program to increase HIV testing uptake among men who have sex with men in Brazil: descriptive cross-sectional analysis. J Med Internet Res. 2019;21:e14145. https://doi.org/10. 2196/14145.

37. Stephenson R, Todd K, Kahle E, Sullivan SP, Miller-Perusse M, Sharma A, et al. Project Moxie: results of a feasibility study of a telehealth intervention to increase HIV testing among binary and nonbinary transgender youth. AIDS Behav. 2020;24:1517-30. https://doi.org/10.1007/s10461-019-02741-z.

38. Wang Z, Lau JTF, Ip M, Ho SPY, Mo PKH, Latkin C, et al. A Randomized Controlled Trial Evaluating Efficacy of Promoting a Home-Based HIV Self-Testing with Online Counseling on Increasing HIV Testing Among Men Who Have Sex with Men. AIDS Behav. 2018;22:190-201. https://doi.org/10.1007/s10461017-1887-2.

39. Wettermann R, Marek H, Giordano TP, Arya M. Text messages can encourage patients to discuss and receive HIV testing in primary care. J Am Board Fam Med. 2019;32:408-12. https://doi.org/10. 3122/jabfm.2019.03.180269.

40. Kelvin EA, George G, Mwai E, Kinyanjui S, Romo ML, Odhiambo JO, et al. A Randomized controlled trial to increase HIV testing demand among female sex workers in Kenya through announcing the availability of HIV self-testing via text message. AIDS Behav. 2019;23:116-25. https://doi.org/10.1007/s10461-018-2248-5. 
41. MacGowan RJ, Chavez PR, Borkowf CB, et al. Effect of internetdistributed HIV self-tests on HIV diagnosis and behavioral outcomes in men who have sex with men: a randomized clinical trial. JAMA Intern Med. 2020;180:117-25. https://doi.org/10.1001/ jamainternmed.2019.5222.

42. Njuguna N, Ngure K, Mugo N, Sambu C, Sianyo C, Gakuo S, et al. The effect of human immunodeficiency virus prevention and reproductive health text messages on human immunodeficiency virus testing among young women in rural Kenya. Sex Transm Dis. 2016:43:353-9. https://doi.org/10.1097/OLQ.0000000000000450.

43. Bauermeister JA, Tingler RC, Demers M, Connochie D, Gillard G, Shaver J, et al. Acceptability and preliminary efficacy of an online HIV prevention intervention for single young men who have sex with men seeking partners online: the myDEx Project. AIDS Behav. 2019;23:3064-77. https://doi.org/10.1007/s10461-019-02426-7.

44. Sullivan PS, Driggers R, Stekler JD, Siegler A, Goldenberg T, McDougal SJ, et al. Usability and acceptability of a mobile comprehensive HIV prevention app for men who have sex with men: a pilot study. JMIR Mhealth Uhealth. 2017;5:e26. https://doi.org/10. 2196/mhealth.7199.

45. Zhu X, Zhang W, Operario D, Zhao Y, Shi A, Zhang Z, et al. Effects of a mobile health intervention to promote HIV selftesting with MSM in China: a randomized controlled trial. AIDS Behav. 2019;23:3129-39. https://doi.org/10.1007/s10461-01902452-5.

46. Horvath KJ, Lammert S, Danh T, Mitchell JW. The feasibility, acceptability and preliminary impact of mobile application to increase repeat HIV testing among sexual minority men. AIDS Behav. 2020;24:1835-50. https://doi.org/10.1007/s10461-019-02755-7.

47. Wray TB, Chan PA, Klausner JD, Mena LA, Brock JB, Simpanen EM, et al. eTest: a limited-interaction, longitudinal randomized controlled trial of a mobile health platform that enables real-time phone counseling after HIV self-testing among high-risk men who have sex with men. Trials. 2020;21:654. https://doi.org/10.1186/ s13063-020-04554-1.

48. Balán IC, Lopez-Rios J, Nayak S, Lentz C, Arumugam S, Kutner B, et al. SMARTtest: A smartphone app to facilitate HIV and syphilis self- and partner-testing, interpretation of results, and linkage to care. AIDS Behav. 2020;24:1560-73. https://doi.org/10.1007/ s10461-019-02718-y.

49. Wray TB, Chan PA, Simpanen E, Operario D. A pilot, randomized controlled trial of HIV self-testing and real-time post-test counseling/referral on screening and preventative care among men who have sex with men. AIDS Patient Care STDs. 2018;32:360-7. https://doi.org/10.1089/apc.2018.0049.

50. Pai NP, Esmail A, Marathe G, et al. Will an app-optimized HIV self-testing strategy work for South Africans? San Fransisco: Results From a Large Cohort Study; 2018. https://watermark. silverchair.com/ofy210.1109.pdf?token=AQECAHi208 B E 49 O o a n $9 \mathrm{k} \mathrm{k} \mathrm{h} \mathrm{W} \mathrm{E} \mathrm{r} \mathrm{c} \mathrm{y} 7 \mathrm{Dm} 3 \mathrm{Z} \mathrm{L}$ 9 C f 3 q fK A c 485 y g A A A t M w g g L P B g k q h k i G9w0BBwagggLAMIICvAIBADCCArUGCSqGSIb3DQ EHATAeBglghkgBZQMEAS4wEQQM4cynyjgp-v $4 \mathrm{~W}$ W O 0 A g E Q g I C hig k E p 1 a U i a z R Y n W g J b n GWTKx8K1OEmdmrCidmM8bStD0SMoJjGZoPEbfsQQ tjelQQrHVJBHtiE3gR2TBlvLN5O63gpPCzQEbuLoP0C T S d Q c z 2 - j 9 A U G H M b j r Y i k K X 7 C k H T L u o 0 e 3 I C m R q J z N A 7 Z Q Z p D w $1 \mathrm{rvgb}$ Q 2 L L YqTNzkS 8D 6tUmVGJGWFy 93 V 8 HnsmX2ZNt_ o h r 4 p B u Q 12 X N y 9 E 5 b H i m v

AzMEK2iZAMhwt8OFdbLLBqfKOwS9h7nBIAj51_ef84H F c N K t E C U O p J U b N r 0 O k h p c Q - 9 Jmzz42hTKPfTeBoWy6HYR4zolftciOw-silbjVh5obSfD_N z W c p 22 i V k 8 d $100-A$ h n R j h R o Z 6 M u $2 \bar{d}$ $82 \mathrm{C} \mathrm{Z} \mathrm{T} \mathrm{H} \mathrm{th} 2$ E z I b 33 y IKR B E S 6 J N n q Z u t $0 \mathrm{I} \mathrm{J} \mathrm{f} 1 \mathrm{M} 7 \mathrm{C}$ Q $1 \mathrm{G} \mathrm{S} \mathrm{Z} \mathrm{c} \mathrm{h} \mathrm{p} \mathrm{Z} \mathrm{Z} 0$ g U y y
Y 6 A t 3 Q R K v j u 5 v h E Y W t 3 t n C L H f S U Iu 44 k Y E R d h 4 V o k 1 x v M Q O n R H 9 a s J k h dP57AmQtwM3CuDnG9XawehuXMhmCjtn44ex3Tfp2dA nQnAmQCEtNO5GZFYWaPuYAVg2jBymVVzamjq9Rr_ s4iMsJnrmlSBcbYRFwU9EwYaFqrOPAxTR98h06EBy Enaskproe9N-_kxXX8D1r6TmgUIHTFzgjfXm O Wn s q A R d f u g k r N 7 w T 7 c w r S R B Uh 76 e P 2 $\mathrm{N}$ T v b n T 3 D O N u P t 0 wi 2 h N y 78 O p J S j 9 rt 4 m B fjg X N L O N 2 ck F B M h 1 S X x 8 X V 9 j r B R B L M h r T R f i t S Y Q j $3 \mathrm{k}$ E JWW H i r w L ex U g x A w b R i OXfZ N q Z z $\overline{7}$ W zJXPZcNECALh81rUKZ3xIU956Dj81XdUbNQ2_ieaueZst7ziY0sF8LPsBjooZCTz8fgiroQSm4mJE2E (accessed 19 Oct 2020)

51. Aronson ID, Bennett A, Marsch LA, Bania TC. Mobile technology to increase $\mathrm{HIV} / \mathrm{HCV}$ testing and overdose prevention/response among people who inject drugs. Front Public Health. 2017;5:217. https://doi.org/10.3389/fpubh.2017.00217.

52. Rosengren AL, Huang E, Daniels J, et al. Feasibility of using GrindrTM to distribute HIV self-test kits to men who have sex with men in Los Angeles, California. Sex Health Published Online First: 23 May 2016. doi:https://doi.org/10.1071/SH15236

53. Aronson ID, Cleland CM, Perlman DC, Rajan S, Sun W, Bania TC. Feasibility of a computer-based intervention addressing barriers to HIV testing among young patients who decline tests at triage. $J$ Health Commun. 2016;21:1039-45. https://doi.org/10.1080/ 10810730.2016.1204382.

54. Tang W, Han L, Best J, Zhang Y, Mollan K, Kim J, et al. Crowdsourcing HIV test promotion videos: a noninferiority randomized controlled trial in China. Clin Infect Dis. 2016;62:143642. https://doi.org/10.1093/cid/ciw171.

55. Kershaw C, Taylor JL, Horowitz G, Brockmeyer D, Libman H, Kriegel G, et al. Use of an electronic medical record reminder improves HIV screening. BMC Health Serv Res. 2018;18:14. https:// doi.org/10.1186/s12913-017-2824-9.

56. Sha BE, Kniuksta R, Exner K, Kishen E, Shankaran S, Williams B, et al. Evolution of an electronic health record based-human immunodeficiency virus (HIV) screening program in an urban emergency department for diagnosing acute and chronic HIV infection. $J$ Emerg Med. 2019;57:732-9. https://doi.org/10.1016/j.jemermed. 2019.08.008.

57. Marcus JL, Hurley LB, Krakower DS, Alexeeff S, Silverberg MJ, Volk JE. Use of electronic health record data and machine learning to identify candidates for HIV pre-exposure prophylaxis: a modelling study. Lancet HIV. 2019;6:e688-95. https://doi.org/10.1016/ S2352-3018(19)30137-7.

58. Ahlström MG, Ronit A, Omland LH, Vedel S, Obel N. Algorithmic prediction of HIV status using nation-wide electronic registry data. EClinicalMedicine. 2019;17:100203. https://doi.org/10.1016/j. eclinm.2019.10.016.

59. Ybarra ML, Prescott TL, Phillips GL, et al. Pilot RCT Results of an mHealth HIV Prevention Program for sexual minority male adolescents. Pediatrics. 2017;140:e20162999. https://doi.org/10.1542/ peds.2016-2999.

60. Kelvin EA, George G, Kinyanjui S, Mwai E, Romo ML, Oruko F, et al. Announcing the availability of oral HIV self-test kits via text message to increase HIV testing among hard-to-reach truckers in Kenya: a randomized controlled trial. BMC Public Health. 2019;19: 7. https://doi.org/10.1186/s12889-018-6345-1.

61. The Safer Sex App. SAFELY. https://safely.me (accessed 21 Oct 2020).

62. Soliz S, Young SD. Feasibility of a citizen-driven hackathon to increase public engagement and solutions to address the opioid crisis. J Subst Use. 2020;25:6, 615-20. https://doi.org/10.1080/ 14659891.2020 .1753833 . 
63. Raffe S, Pollard A, Vera JH, Soni S, Peralta C, Rodriguez L, et al. HIV self-tests for men who have sex with men, accessed via a digital vending machine: a qualitative study of acceptability. Int $J$ STD AIDS. 2020;31:420-5. https://doi.org/10.1177/ 0956462419890726.

64. Lee MJ, Onyango D, Hamza H, Phiri E, Furlong T, Goel P, et al. Surveying testing preferences in Black, Latin American, and other minorities for the co-design of digital vending machines for HIV self-testing. Int J STD AIDS. 2020;31:158-65. https://doi.org/10. $1177 / 0956462419887042$.

65. Stafylis C, Natoli LJ, Murkey JA, et al. Vending machines in commercial sex venues to increase HIV self-testing among men who have sex with men. Mhealth. 2018;4:51. https://doi.org/10.21037/ mhealth.2018.10.03.

66. He X, Feng X, Liu P, Lyu Y, Lu H, Ma Y, et al. An innovative vending machine-based HIV testing and intervention service in China: anonymous urine collection kits distributed at universities. Aids Care-Psychol Socio-Med Asp Aids-Hiv. 2019;31:1319-22. https://doi.org/10.1080/09540121.2019.1612012.

67. Vera JH, Soni S, Pollard A, Llewellyn C, Peralta C, Rodriguez L, et al. Acceptability and feasibility of using digital vending machines to deliver HIV self-tests to men who have sex with men. Sex Transm Infect. 2019;95:557-61. https://doi.org/10.1136/sextrans2018-053857.

68. Young SD, Daniels J, Chiu CJ, et al. Acceptability of using electronic vending machines to deliver oral rapid HIV self-testing kits: a qualitative study. PLoS One. 2014;9:e103790. https://doi.org/10. 1371/journal.pone.0103790.

69. Brixey J, Hoegen R, Lan W, et al. SHIHbot: A Facebook chatbot for sexual health information on HIV/AIDS. In: Proceedings of the 18th Annual SIGdial Meeting on Discourse and Dialogue. Saarbrücken, Germany: : Association for Computational Linguistics 2017. 370-3. doi:https://doi.org/10.18653/v1/W175544

70. van Heerden A, Ntinga X, Vilakazi K. The potential of conversational agents to provide a rapid HIV counseling and testing services. In: 2017 International Conference on the Frontiers and Advances in Data Science (FADS). 2017. 80-5. doi:https://doi.org/10.1109/ FADS.2017.8253198

71. Vermey K, Daas CD, Zweers W, et al. P046 Ensuring qualityassured and personalized online self-testing within a marketdriven context. In: July 15, 2019. Vancouver, Canada: : BMJ Publishing Group Ltd 2019. https://sti.bmj.com/content/95/Suppl_ 1/A99.1 (accessed 19 Oct 2020).

72. UNAIDS. Chatbot answers young people's questions about HIV, health and relationships. UNAIDS.org. 2020.https://www.unaids. org/en/resources/presscentre/featurestories/2020/october/ 20201015_chatbot (accessed 19 Oct 2020).

73. Tankovska H. Level of usage of voice assistants across various functions worldwide, as of November 2017. Statista. 2020.https:// www.statista.com/statistics/801963/worldwide-level-usage-voiceassistant-various-functions/ (accessed 30 Oct 2020).

74. Muessig KE, Knudtson KA, Soni K, Larsen MA, Traum D, Dong W, et al. "I didn't tell you sooner because i didn't know how to handle it myself." Developing a virtual reality program to support HIV-status disclosure decisions. Digit Cult Educ. 2018;10:22-48.

75. Liran O, Dasher R, Kaeochinda K. Using virtual reality to improve antiretroviral therapy adherence in the treatment of HIV: open-label repeated measure study. Interact J Med Res. 2019;8:e13698. https:// doi.org/10.2196/13698.

76. Pilkington M. Blockchain technology: principles and applications. Rochester, NY: : Social Science Research Network 2015. https:// papers.ssrn.com/abstract=2662660 (accessed 5 Nov 2020).

77. Underwood S. Blockchain beyond bitcoin. Commun ACM. 2016;59:15-7. https://doi.org/10.1145/2994581.

78. Yli-Huumo J, Ko D, Choi S, Park S, Smolander K. Where is current research on blockchain technology? - a systematic review. PLoS One. 2016;11:e0163477. https://doi.org/10.1371/journal.pone. 0163477.

79. Dib O, Brousmiche K-L, Durand A, et al. Consortium blockchains: overview, applications and challenges. International Journal On Advances in Telecommunications Published Online First: 2018.https://hal.archives-ouvertes.fr/hal-02271063 (accessed 5 Nov 2020).

80. Statista. Mobile payment usage in the United States. https://www. statista.com/study/11648/mobile-payments-statista-dossier/ (accessed 21 Oct 2020).

81. J.P. Morgan Merchant Services. Digital Payments are Redefining POS. J.P. Morgan. https://www.jpmorgan.com/merchant-services/ insights/digital-payments-are-redefining-pos (accessed 21 Oct 2020).

82. Garett R, Menacho L, Young SD. Ethical issues in using social media to deliver an HIV prevention intervention: results from the HOPE Peru Study. Prev Sci. 2017;18:225-32. https://doi.org/10. 1007/s11121-016-0739-z.

83. Romero RA, Young SD. Ethical views on sharing digital data for public health surveillance: a qualitative analysis of survey data among patients. In press 2021.

84. Young SD, Schneider J. Clinical care, research, and telehealth services in the era of social distancing to mitigate COVID-19. AIDS Behav 2020;:1-3. doi:https://doi.org/10.1007/s10461-020-02924-Z

85. Young SD. The Adaptive Behavioral Components (ABC) Model for planning longitudinal behavioral technology-based health interventions: a theoretical framework. J Med Internet Res. 2020;22: e15563. https://doi.org/10.2196/15563.

86. Li DH, Brown CH, Gallo C, Morgan E, Sullivan PS, Young SD, et al. Design considerations for implementing eHealth behavioral interventions for HIV prevention in evolving sociotechnical landscapes. Curr HIV/AIDS Rep. 2019;16:335-48. https://doi.org/10. 1007/s11904-019-00455-4.

87. Young SD. Stick with it: a scientifically proven process for changing your life-for good. Harper Collins

Publisher's Note Springer Nature remains neutral with regard to jurisdictional claims in published maps and institutional affiliations. 